\title{
Combination chemotherapy of cancer using the inhibitor of DNA methylation 5-aza-2'-deoxycytidine (decitabine)
}

\author{
Leen Stephan ${ }^{1}$ and Richard L Momparler ${ }^{1,2, *}$ \\ 1 Département de Pharmacologie; Université de Montréal, Montréal, Québec, Canada \\ 2 Service of Hematology and Oncology, Centre de recherche, CHU-Saint-Justine, 3175 Côte Sainte-Catherine, Montréal, Québec H3T 1C5, Canada
}

\begin{abstract}
The epigenetic alterations marked by DNA methylation contribute to the malignant transformation of cells by silencing critical genes responsible for the regulation of growth. The potent DNA methylation inhibitor 5-aza-2'-deoxycytidine (decitabine; DAC) has shown effectiveness in patients with myeloid malignancies. However, the responses are of short duration. The effectiveness of the DAC therapy may be limited by its incapacity to reactivate enough tumor suppressor genes. Other epigenetic mechanisms, such as the histone modification of target genes, may also hinder gene reactivation by DAC. The dose limiting toxicity of DAC is myelosuppression, which limits the duration of this therapy for clinical use. The clinical effectiveness of DAC may be enhanced by its use in combination with other agents that have diverse mechanisms of action. In this literature review, we summarize the results of preclinical and recent clinical trials of DAC used in combination with other agents to treat cancer. This review was conducted by searching online databases to analyze the available evidence regarding this area of interest. We looked at the combination of DAC with other epigenetic agents, cytotoxic agents, tyrosine kinase inhibitors, biochemical modulators and non-toxic agents. The data compiled suggests that combination epigenetic therapy is feasible, moderately toxic and has promising clinical potential. Preclinical studies showed that some combinations of DAC have additive to synergistic antineoplastic action as compared to DAC alone. The data indicate that combination chemotherapy with DAC merits further investigation. This review may be helpful for the future design of clinical trials using DAC in combination for cancer therapy.
\end{abstract}

Keywords: decitabine; 5-aza-2'-deoxycytdine; epigenetics; acute myeloid leukemia; cancer; combination chemotherapy; DNA methylation; epigenetic agents; histone deacetylase inhibitors; tyrosine kinase inhibitors; non-toxic agents

\section{Introduction}

Epigenetic modifications play a critical role in the development of different types of cancer by contributing to the cumulative changes in normal cells undergoing malignant transformation. These modifications have been used to develop epigenome-targeted therapies and new diagnostic tools. Since the silencing of tumor suppressor genes by epigenetic changes is reversible, it is a promising drug target for suppressing malignant growth and restoring the normal cellular phenotype [1]. These epigenetic changes that silence tumor suppressor genes (TSGs) can involve aberrant DNA methylation and/or changes in chromatin structure [2]. The hypermethylation of $\mathrm{CpG}$ rich islands within the promoter region of certain tumor suppressor genes is a hallmark of malignancy [3].

5-aza-2'-deoxycytidine (decitabine; DAC), an analog of deoxycytidine, contains nitrogen substituted for carbon at position 5 of the pyrimidine ring [4]. DAC is a prodrug that requires metabolic activation by deoxycytidine kinase, an active inhibitor in the triphosphate form [1]. DNA polymerase catalyzes the insertion of the phosphorylated form of DAC into DNA. The presence of DAC in place of the 5-methylcytosine in DNA leads to the inactivation of DNA methyltransferase (DNMT), which causes the demethylation of DNA and induces a re-expression of the silenced genes [2].

In clinical practice, DAC is an effective therapy for myelodysplastic syndromes (MDS) and for acute myeloid leukemia (AML) [5]. Although effective, DAC monotherapy has been associated with short durations of complete remission (CR) rates in MDS and AML. In a phase III randomized study of DAC on 170 MDS patients, the overall response rate (OR) was 17\% including 9\% CR [6]. Furthermore, in a phase I study of 37 patients with AML receiving DAC, the OR was $17 \%$ [7]. The dose limiting toxicity

\footnotetext{
*Corresponding author: Richard L Momparler, Service of Hematology and Oncology, Centre de recherche, CHU-Saint-Justine, 3175 Côte SainteCatherine, Montréal, Québec H3T 1C5, Canada. Tel.: 514 345-4931, poste 6140; Fax: 514 345-4801; Email: richard.I.momparler@umontreal.ca

Received 15 March 2015 Revised 2 May 2015 Accepted 15 May 2015 Published 25 May 2015

Citation: Stephan L, Momparler RL. Combination chemotherapy of cancer using the inhibitor of DNA methylation 5-aza-2'-deoxycytidine (decitabine). J Cancer Res Ther. 2015; 3(5):56-65. DOI:10.14312/2052-4994.2015-8

Copyright: ( 2015 Stephan L, et al. Published by NobleResearch Publishers. This is an open-access article distributed under the terms of the Creative Commons Attribution License, which permits unrestricted use, distribution and reproduction in any medium, provided the original author and source are credited.
} 
of DAC was myelosuppression [8]. This toxicity is reversible and additional cycles of DAC can be administered every 4 to 6 weeks. The toxicity of DAC plus the limited demethylation in malignant cells and the eventual remethylation of genes may limit its effectiveness. Two major challenges limit the clinical use of DAC: first, the need to increase its antineoplastic action without increasing its hematopoietic toxicity and second, the problem of drug resistance.

The initial clinical studies on DAC showed marked improvement in patients with MDS [6]. An elucidation of the different mechanisms of gene silencing has led to an interest in combining drugs that affect multiple epigenetic pathways [9]. DNA methylation and repressive histone marks in chromatin work in a positive manner to silence gene expression [10]. After the methylation of $\mathrm{CpGs}$ in the promoter region, methyl-CpG binding domain (MBD) proteins attach to this target and recruit histone deacetylase (HDAC) complexes. This epigenetic silencing of gene expression is a result of the blockage of the attachment of transcription factors and the compaction of chromatin due to HDAC [11]. The interaction between these two epigenetic events is illustrated by reports that HDAC inhibitors, such as trichostatin A, phenylbutyrate and depsipeptide enhance the in vitro activation of tumor suppressor genes by DAC and its antineoplastic activity $[9,12]$. Depsipeptide (Istodax ${ }^{\circledR}$ ) and vorinostat (Zolinza ${ }^{\circledR}$ ) are two HDAC inhibitors that have been approved for the treatment of cutaneous T-cell lymphoma (CTCL); both drugs appear to inhibit multiple HDAC classes. Depsipeptide is a prodrug that undergoes conversion to its reduced active form, which then interacts with the binding pocket and inhibits several HDACs. Vorinostat, a linear hydroxamate, also appears to inhibit multiple classes of HDAC [13]. Another potential obstacle for curative therapy with DAC is the presence of very high levels of cytidine deaminase (CD), a key enzyme in the metabolism of DAC. Rapid inactivation of DAC by CD could result in a complete loss of its antineoplastic activity. Zebularine (Zeb) is an inhibitor of $C D$ that is capable of blocking the deamination of DAC [14]. It also has its own antineoplastic activity due to its weak inhibition of DNMT [15]. These characteristics make this agent appealing for investigation in combination with DAC.

An interesting agent to overcome drug-resistance is 3-deazauridine (3-DU). Leukemic cells deficient in deoxycytidine kinase(DCK) arevery sensitive to the cytotoxic action of 3-DU [16]. The active phosphorylated form of 3-DU inhibits CTP synthetase and reduces the intracellular level of CTP and dCTP. Due to the competition of DAC triphosphate and dCTP for DNA polymerase, a reduction in dCTP levels result in an increased incorporation of DAC into DNA and an enhancement of its antineoplastic action [17].

Another agent worth studying in combination with DAC is 3-deazaneplanocin-A (DZNep), an inhibitor of histonelysine $\mathrm{N}$-methyltransferase (EZH2). EZH2 is a subunit of the polycomb repressive complex 2 and catalyzes the trimethylation of histone 3 lysine 27, a marker for gene silencing. A "cross-talk" exists between EZH2 and DNA methylation, which is caused by the recruitment of DNMTs by $\mathrm{EZH} 2$ to the promoter region of the target genes [18].
Some tyrosine kinase inhibitors (TKI) can block the signal transduction pathway involved in the proliferation and survival of cancer cells. Gefitinib is an orally selective epidermal growth factor receptor (EGFR)-TKI with a demonstrated antitumor activity in solid tumors [19]. It has been shown that gefitinib can abate chemotherapy resistance by inhibiting the transmembrane transporters of the ABC family, including P-glycoprotein (P-gp) and the multidrug resistance protein 1 (MRP1), making gefitinib an interesting target for use in combination regimens [20].

Non-toxic agents can help sensitize cells for epigenetic agents and serve as adjuvants in chemotherapy of cancer. For example, retinoids have been shown to induce in vitro differentiation and to inhibit the growth of different types of neoplastic cells [21]. Retinoic acid exhibits interesting antineoplastic activity and has induced complete remissions in patients with acute promelocytic leukemia [22]. Genistein, a non-toxic soybean isoflavone, has been reported to have a chemopreventitive and anticancer activity, as well as an ability to enhance the activity of some cytotoxic antineoplastic drugs [23]. Combining these agents could increase the effectiveness of chemotherapy when used in combination with DAC. Another non-toxic agent to investigate is vitamin $D$, since it also has been reported to induce the in vitro differentiation of human myeloid leukemia cell lines. Several analogs of vitamin D have been synthesized with reduced activity in calcium metabolism, but still retain the ability to induce leukemic cell differentiation, although the calcium toxicity produced by vitamin $D$ limits its clinical use for leukemia therapy [24].

In this review, we sought to compare the effectiveness of different combination regimens of DAC in preclinical studies, as well as their tolerability in clinical trials relative to the use of DAC alone.

\section{Materials and methods}

\section{Data sources and searches}

The research strategy for this review consisted of conducting a search for all articles in MEDLINE via PubMed and Google Scholar with publication dates from 2000 to November 2014. The search included the following terms: decitabine, combination, HDAC, and AML. A search for decitabine and combination on PubMed yielded approximately 400 results. Specific searches also were done with different drug names in combination with DAC, for example, with vorinostat, trichostatin $A$, and valproic acid. In general, we searched the electronic databases for preclinical (in vivo and in vitro) and clinical trials that used DAC in combination with other agents to treat hematologic malignancies and solid tumors. We searched PubMed for reports of clinical trials by adding phase and/or clinical to the search. We searched http://www.clinicaltrials.gov to identify active or recently completed trials of the same interventions. We also looked at reference lists of relevant articles and reviews.

\section{Study selection}

After potentially relevant studies were found, they were screened for specific relevance to our review by referring 
to their title, abstract, and the full text article. Of the 400 articles and abstracts identified by the search, about 60 articles initially met the criteria for inclusion. These were assessed with regard to the following general inclusion criteria: peer-reviewed, English language articles only, with relevance to the research topic. Reviews, meta-analyses, case reports and letters were excluded from further analysis. Studies with only published abstracts were excluded. More specific exclusion criteria were: the use of DAC in combination with two or more drugs, or the use of azacitidine (5-azacytidine) - another DNA methylating agent - instead of decitabine in combination regimens. Relevant articles included 26 in vitro studies and 11 in vivo studies. Study results for preclinical studies were included in the case of synergistic or positive effects, and were excluded in the case of a statistically insignificant effect. Relevant articles also included 7 phase I and II studies. The quality of all individual studies was evaluated based on sound experimental design, and the studies were rated as good, fair, or poor based on their adherence to wellaccepted standard methodologies and adequate reporting. A few articles published prior to 2000 were added during the search, since they were thought to be worthy of further investigation.

\section{Data extraction and quality assessment}

\section{In vitro}

In this literature review, 26 in vitro studies were assessed. A clonogenic (colony) cell survival assay was used in most of the studies to determine the ability of neoplastic cells to proliferate. This assay is used to examine the effects of an antineoplastic agent on cell survival. A cell survival curve reflects the relationship between dose of the agent used to produce an insult and the fraction of cells retaining their ability to reproduce. The mechanism by which DAC produces a loss of clonogenicity in leukemic cells is most likely related to its activation of genes that have been silenced by aberrant DNA methylation and whose roles are to program terminal differentiation, apoptosis, or senescence [5]. The treatment of leukemic cell lines with DAC has been shown to reactivate several different tumor suppressor genes that were silenced by epigenetic events. Survival curves have been generated for many established cell lines growing in culture. In the studies assessed, cell lines were used from various origins.

Cell lines in these studies were treated with DAC and the agent to be studied in combination with it separately and then compared to combination with both treatments. The treatment was either simultaneous - as with most of the 26 studies assessed - or sequential. Usually, sequential treatment is carried out with DAC first, followed by the other agent combined with DAC. In the article, if the percentage loss of clonogenicity was not provided numerically as a percentage, it was either approximated from the survival fraction or read off the cell survival curve. The interaction was described as synergistic when SF = Survival fraction, SF drug A + SF drug D < SF drug A X SF drug D [Valeriote and Lin] [25]. In the studies assessed, cell viability and apoptotic assays also were used occasionally to study the effects of the neoplastic and cytotoxic agents on different cell lines.

\section{In vivo}

The antitumor effect in animal models ofDAC in combination with other agents was ascertained by observing the changes in body weight, tumor volume, mean survival time (MST) and percentage increase in life span (\%ILS). The MST was noted with reference to the control. The MTS of treated groups $(T)$ was compared with those of control groups (C) using the following equation: Increase of life span = T - C / $\mathrm{C} \times 100$. The percentage increase in lifespan was calculated using the following formula:

ILS \% $=($ MST of treated group/ MST of control group -1$) \times 100$.

\section{Clinical}

A total of 7 clinical trials, consisting of phase I and phase I/ II studies of various doublet combinations, were examined to provide further evidence of the potential use of combination therapy in a clinical setting. Different tests were used to assess the severity and outcome of cancer, which included but were not limited to the following: Complete remission (CR), Partial remission (PR), Overall response rate (ORR), and Hematologic improvement $(\mathrm{HI})$. All data extracted and approximated from the articles is presented in tables and sorted according to drug class and type of cancer (hematologic versus solid tumor).

\section{Results}

Tables from 1 to 5 present the additive or synergistic effect for the activity of DAC when combined with different agents and assessed experimentally in cell lines. For the selected in vitro studies the combination of the two agents gives a greater reduction in colony formation or percentage of cell viability than with either agent alone. Tables ( 6 and 7 ) also present some notable synergistic effects for the use of some agents in combination with DAC in animal studies as reflected in the assessment of tumor volume or the percentage increase in life span (\% ILS) in mouse models. Tables ( 8 and 9) present select clinical trials that assess the use of DAC in combination therapy.

\section{Combinations in preclinical studies}

\section{Histone deacetylase inhibitors (HDAC-I)}

Panobinostat: Panobinostat, a cinnamic hydroxamic acid analog, is an inhibitor that targets a broad spectrum of HDACs including class I and class II enzymes [26]. A DAC treatment combined with panobinostat inhibits clonogenic survival to a greater extent than treatment with a single agent. $\mathrm{K} 562$ leukemic cells were treated with the concentrations indicated in Table 1 for 48 hours, and the combined activity decreased the colony growth of these cells by about $80 \%$ as compared to $<50 \%$ for each agent alone [27]. In another study, the efficacy of panobinostat in combination with DAC was evaluated in lymphoma cell lines and a tumor xenograft model of diffuse large B-cell lymphoma (DLBCL). Experiments were performed in Ly1 and Ly10 DLBCL lines; DAC plus panobinostat was shown to have a synergistic effect (Table 1). Synergy was validated in a number of other assays, including caspase 3 activation and apoptosis. The in vitro observations also were confirmed by a murine xenograft in vivo experiment with the Ly1 DLBCL line [28]. These data demonstrated that the combination of panobinostat and DAC was synergistically 
Table 1 In vitro antineoplastic action of decitabine (DAC) in combination with HDAC inhibitors for the treatment of hematologic malignancies.

\begin{tabular}{|c|c|c|c|c|c|c|c|c|}
\hline Cancer & Cell Line & $\begin{array}{l}\text { DAC/ Dose } \\
\text { schedule }\end{array}$ & $\begin{array}{c}D A C \\
\text { activity }\end{array}$ & $\begin{array}{c}\text { HDAC } \\
\text { inhibitor }\end{array}$ & $\begin{array}{l}\text { HDAC/ Dose } \\
\text { schedule }\end{array}$ & $\begin{array}{c}H D A C \\
\text { activity }\end{array}$ & $\begin{array}{c}\text { Combination } \\
\text { activity }\end{array}$ & Ref. \\
\hline $\mathrm{AML}^{*}$ & K562 & $2 \mu \mathrm{M}, 48 \mathrm{~h}$ & $\downarrow \sim 30 \%$ colony & Panobinostat & $10 \mathrm{nM}, 48 \mathrm{~h}$ & $\downarrow \sim 50 \%$ colony & $\downarrow \sim 80 \%$ colony & [27] \\
\hline AML & HL-60 & $0.04 \mu \mathrm{M}, 72 \mathrm{~h}$ & $\downarrow 59 \%$ colony & Depsipeptide & $1.4 \mathrm{nM}, 72 \mathrm{~h}$ & $\downarrow 65 \%$ colony & $\downarrow 90 \%$ colony & [33] \\
\hline AML & KASUMI- 1 & $2.5 \mu \mathrm{M}, 48 \mathrm{~h}$ & $\downarrow 15 \%$ viable cells & Depsipeptide & $10 \mathrm{nM}, 48 \mathrm{~h}$ & $\downarrow 75 \%$ viable cells & $\downarrow 90 \%$ viable cells & {$[65]$} \\
\hline AML & HL-60 & $0.09 \mu \mathrm{M}, 96 \mathrm{~h}$ & $\downarrow 43 \%$ colony & Phenyl-butyrate & 0.5 mM, 96h & $\downarrow 25 \%$ colony & $\downarrow 62 \%$ colony & {$[12]$} \\
\hline AML & HL-60 & $0.04 \mu \mathrm{M}, 72 \mathrm{~h}$ & $\downarrow 59 \%$ colony & Trichostatin-A & $16.6 \mathrm{nM}, 72 \mathrm{~h}$ & $\downarrow 15 \%$ colony & $\downarrow 91 \%$ colony & {$[33]$} \\
\hline AML & HL-60 & $0.02 \mu \mathrm{M}, 24 \mathrm{~h}$ & $\downarrow \sim 20 \%$ colony & Trichostatin-A & $80 \mathrm{nM}, 24 \mathrm{~h}$ & $\downarrow \sim 20 \%$ colony & $\downarrow \sim 50 \%$ colony** & {$[46]$} \\
\hline AML & AML-3 & $0.02 \mu \mathrm{M}, 24 \mathrm{~h}$ & $\downarrow \sim 55 \%$ colony & Trichostatin-A & $40 \mathrm{nM}, 24 \mathrm{~h}$ & $\downarrow \sim 45 \%$ colony & $\downarrow \sim 80 \%$ colony** & [46] \\
\hline Lymphoma & Raji & $0.01 \mu \mathrm{M}, 72 \mathrm{~h}$ & $\downarrow 28 \%$ colony & Despsipeptide & $1.4 \mathrm{nM}, 48 \mathrm{~h}$ & $\downarrow 27 \%$ colony & $\downarrow 81 \%$ colony** & [32] \\
\hline Lymphoma & L1210 & $0.004 \mu \mathrm{M}, 48 \mathrm{~h}$ & $\downarrow 41 \%$ colony & Phenylbutyrate & $1 \mathrm{mM}, 48 \mathrm{~h}$ & $\downarrow 56 \%$ colony & $\downarrow 89 \%$ colony & {$[12]$} \\
\hline $\mathrm{HTCL}^{*}$ & MOLT4 & $1 \mu \mathrm{M} \times 4 \mathrm{~d}$ & $\downarrow 65 \%$ viable cells & Valproic Acid & $1 \mathrm{mM} \times 3 \mathrm{~d}$ & $\downarrow 53 \%$ viable cells & $\downarrow 90 \%$ viable cells ** & {$[30]$} \\
\hline
\end{tabular}

Abbreviations: *AML= acute myeloid leukemia; $\mathrm{DLBCL}=$ diffuse large B-cell lymphoma; HTCL= human T-cell leukemia; ** sequential treatment (DAC followed by the other agent); [Conversion factor, $228 \mathrm{ng} / \mathrm{ml}=1 \mu \mathrm{M}$ decitabine, $10^{-6} \mathrm{M}=1 \mu \mathrm{M}$ ].

Table 2 In vitro antineoplastic action of decitabine (DAC) in combination with HDAC inhibitors for the treatment of solid tumors.

\begin{tabular}{|c|c|c|c|c|c|c|c|c|}
\hline Cancer & Cell Line & $\begin{array}{l}\text { DAC/ Dose } \\
\text { schedule }\end{array}$ & $\begin{array}{c}D A C \\
\text { activity }\end{array}$ & $\begin{array}{c}\text { HDAC } \\
\text { inhibitor }\end{array}$ & $\begin{array}{l}\text { HDAC/ Dose } \\
\text { schedule }\end{array}$ & $\begin{array}{l}\text { HDAC } \\
\text { activity }\end{array}$ & $\begin{array}{c}\text { Combination } \\
\text { activity }\end{array}$ & Ref. \\
\hline Ovarian & Hey & $10 \mu \mathrm{M}, 24 \mathrm{~h}$ & $\downarrow \sim 40 \%$ colony & SAHA* & $0.5 \mu \mathrm{M}, 24 \mathrm{~h}$ & $\downarrow \sim 20 \%$ colony & $\downarrow \sim 85 \%$ colony & [41] \\
\hline Ovarian & SKOv3 & $10 \mu \mathrm{M}, 24 \mathrm{~h}$ & $\downarrow \sim 50 \%$ colony & SAHA* & $0.5 \mu \mathrm{M}, 24 \mathrm{~h}$ & $\downarrow \sim 20 \%$ colony & $\downarrow \sim 75 \%$ colony & [41] \\
\hline Ovarian & Hey & $10 \mu \mathrm{M}, 24 \mathrm{~h}$ & $\downarrow 45 \%$ colony & Trichostatin-A & $30 \mathrm{nM}, 24 \mathrm{~h}$ & $\downarrow 10 \%$ colony & $\downarrow 75 \%$ colony & [41] \\
\hline Ovarian & SKOv3 & $10 \mu \mathrm{M}, 24 \mathrm{~h}$ & $\downarrow 40 \%$ colony & Trichostatin-A & $30 \mathrm{nM}, 24 \mathrm{~h}$ & $\downarrow 20 \%$ colony & $\downarrow 70 \%$ colony & [41] \\
\hline Pancreatic & PANC-1 & $1 \mu \mathrm{M}, 96 \mathrm{~h}$ & $\downarrow \sim 30 \%$ viable cells & $\mathrm{SAHA} *$ & $2.5 \mu \mathrm{M}, 96 \mathrm{~h}$ & $\downarrow \sim 40 \%$ viable cells & $\downarrow \sim 70 \%$ viable cells & {$[42]$} \\
\hline Pancreatic & AsPC-1 & $1 \mu \mathrm{M}, 96 \mathrm{~h}$ & $\downarrow \sim 25 \%$ viable cells & SAHA* & $2.5 \mu \mathrm{M}, 96 \mathrm{~h}$ & $\downarrow \sim 60 \%$ viable cells & $\downarrow \sim 80 \%$ viable cells & {$[42]$} \\
\hline EWS * & TC71 & $0.04 \mu \mathrm{M}, 48 \mathrm{~h}$ & $\downarrow \sim 20 \%$ colony & MS-275 & $0.27 \mu \mathrm{M}, 48 \mathrm{~h}$ & $\downarrow \sim 10 \%$ colony & $\downarrow \sim 75 \%$ colony & {$[66]$} \\
\hline EWS * & TC71 & $0.04 \mu \mathrm{M}, 48 \mathrm{~h}$ & $\downarrow \sim 20 \%$ colony & LAQ824 & $13 \mathrm{nM}, 48 \mathrm{~h}$ & $\downarrow \sim 20 \%$ colony & $\downarrow \sim 78 \%$ colony & {$[66]$} \\
\hline NSCLC* & A549 & $0.22 \mu \mathrm{M}, 72 \mathrm{~h}$ & $\downarrow 50 \%$ colony & Phenyl-Butyrate & $2 \mathrm{mM}, 72 \mathrm{~h}$ & $\downarrow 25 \%$ colony & $\downarrow 85 \%$ colony & {$[36]$} \\
\hline Breast & Hs578T & $0.15 \mu \mathrm{M}, 48 \mathrm{~h}$ & $\downarrow \sim 50 \%$ colony & Depsipeptide & $0.93 \mathrm{nM}, 48 \mathrm{~h}$ & $\downarrow \sim 70 \%$ colony & $\downarrow \sim 95 \%$ colony & [34] \\
\hline Breast & MCF-7 & $0.09 \mu \mathrm{M}, 48 \mathrm{~h}$ & $\downarrow \sim 25 \%$ colony & Depsipeptide & $0.74 \mathrm{nM}, 48 \mathrm{~h}$ & $\downarrow \sim 18 \%$ colony & $\downarrow \sim 70 \%$ colony & {$[34]$} \\
\hline Breast & MDA-MB-435 & $0.04 \mu \mathrm{M}, 48 \mathrm{~h}$ & $\downarrow \sim 30 \%$ colony & Depsipeptide & $0.65 \mathrm{nM}, 48 \mathrm{~h}$ & $\downarrow \sim 40 \%$ colony & $\downarrow \sim 65 \%$ colony & [35] \\
\hline
\end{tabular}

Abbreviations: *EWS= advanced ewing's sarcoma; NSCLC= non-small cell lung cancer; SAHA= suberanilohydroxamic acid; ** sequential treatment; [Conversion factor, $228 \mathrm{ng} / \mathrm{ml}=1 \mu \mathrm{M}$ decitabine, $10^{-6} \mathrm{M}=1 \mu \mathrm{M}$ ].

Table 3 In vitro antineoplastic action of decitabine (DAC) in combination with other epigenetic drugs for the treatment of hematologic leukemia.

\begin{tabular}{|c|c|c|c|c|c|c|c|c|}
\hline Cancer & Cell Line & $D A C /$ Dose schedule & $\begin{array}{c}D A C \\
\text { activity }\end{array}$ & Epigenetic drug & $\begin{array}{l}\text { Drug } \\
\text { conc }\end{array}$ & $\begin{array}{l}\text { Drug } \\
\text { activity }\end{array}$ & $\begin{array}{c}\text { Combination } \\
\text { activity }\end{array}$ & Ref. \\
\hline $\mathrm{AML}^{*}$ & HL-60 & $0.04 \mu \mathrm{M}, 48 \mathrm{~h}$ & $\downarrow \sim 51 \%$ colony & Zebularine & $0.44 \mu \mathrm{M}, 48 \mathrm{~h}$ & $\downarrow \sim 27 \%$ colony & $\downarrow \sim 95 \%$ colony & [9] \\
\hline $\mathrm{AML}^{*}$ & HL-60 & $0.01 \mu \mathrm{M}, 48 \mathrm{~h}$ & $\downarrow \sim 38 \%$ colony & DZNep* & $1000 \mathrm{nM}, 48 \mathrm{~h}$ & $\downarrow \sim 35 \%$ colony & $\begin{array}{l}\downarrow \sim 95 \% \\
\text { colony** }\end{array}$ & {$[47]$} \\
\hline $\mathrm{AML}^{*}$ & HL-60 & $0.02 \mu \mathrm{M}, 24 \mathrm{~h}$ & $\downarrow \sim 20 \%$ colony & DZNep* & $1000 \mathrm{nM}, 24 \mathrm{~h}$ & $\downarrow \sim 35 \%$ colony & $\begin{array}{l}\downarrow \sim 62 \% \\
\text { colony** }\end{array}$ & {$[46]$} \\
\hline $\mathrm{AML}^{*}$ & AML-3 & $0.02 \mu \mathrm{M}, 24 \mathrm{~h}$ & $\downarrow \sim 55 \%$ colony & DZNep* & $500 \mathrm{nM}, 24 \mathrm{~h}$ & $\downarrow \sim 20 \%$ colony & $\begin{array}{l}\downarrow \sim 65 \% \\
\text { colony** }\end{array}$ & {$[46]$} \\
\hline $\mathrm{AML}^{*}$ & HL-60 & $0.2 \mu \mathrm{M}, 24 \mathrm{~h}$ & $\downarrow \sim 30 \%$ colony & 3- DU* & $20 \mu \mathrm{M}, 24 \mathrm{~h}$ & $\downarrow \sim 80 \%$ colony & $\downarrow \sim 98 \%$ colony & [16] \\
\hline Leukemia & L1210 & $0.004 \mu \mathrm{M}, 48 \mathrm{~h}$ & $\downarrow \sim 33 \%$ colony & Zebularine & $0.44 \mu \mathrm{M}, 48 \mathrm{~h}$ & $\downarrow \sim 11 \%$ colony & $\downarrow \sim 60 \%$ colony & [9] \\
\hline Leukemia & V5 & $0.09 \mu \mathrm{M}, 48 \mathrm{~h}$ & $\downarrow \sim 9 \%$ colony & Zebularine & $4.4 \mu \mathrm{M}_{\prime \prime} 48 \mathrm{~h}$ & $\downarrow \sim 16 \%$ colony & $\downarrow \sim 45 \%$ colony & {$[44]$} \\
\hline Leukemia & L1210 & $0.1 \mu \mathrm{M}, 48 \mathrm{~h}$ & $\downarrow 52 \%$ colony & DZNep & $100 \mathrm{nM}, 48 \mathrm{~h}$ & $\downarrow 18 \%$ colony & $\downarrow 88 \%$ colony** & {$[47]$} \\
\hline
\end{tabular}

Abbreviations: *AML= acute myeloid leukemia; DZNep: 3-deazaneplanocin A; 3-DU: 3-deazauridine; ** sequential treatment; [Conversion factor, 228 ng/ml = $1 \mu \mathrm{M}$ decitabine, $\left.10^{-6} \mathrm{M}=1 \mu \mathrm{M}\right]$. 
Table 4 In vitro antineoplastic action of decitabine (DAC) in combination with tyrosine kinase inhibitors for the treatment of cancer.

\begin{tabular}{|c|c|c|c|c|c|c|c|c|}
\hline Cancer & Cell Line & $\begin{array}{l}\text { DAC/ Dose } \\
\text { schedule }\end{array}$ & $\begin{array}{c}D A C \\
\text { activity }\end{array}$ & Agent & $\begin{array}{l}\text { Agent } \\
\text { conc }\end{array}$ & $\begin{array}{l}\text { Agent } \\
\text { activity }\end{array}$ & $\begin{array}{c}\text { Combination } \\
\text { activity }\end{array}$ & Ref. \\
\hline $\mathrm{AML}^{*}$ & MV4-11 & $0.01 \mu \mathrm{M}, 24 \mathrm{~h}$ & $10 \%$ apoptosis & Midostaurin & $100 \mathrm{nM}, 24 \mathrm{~h}$ & $40 \%$ apoptosis & $\sim 50 \%$ apoptosis & [49] \\
\hline $\mathrm{AML}^{*}$ & MOLM13 & $0.01 \mu \mathrm{M}, 24 \mathrm{~h}$ & $45 \%$ apoptosis & Midostaurin & $100 \mathrm{nM}, 24 \mathrm{~h}$ & $\sim 12 \%$ apoptosis & $\sim 75 \%$ apoptosis & {$[49]$} \\
\hline Colon & SW1116 & $10 \mu \mathrm{M}, 48 \mathrm{~h}$ & $\downarrow \sim 10 \%$ colony & Gefitinib & $2 \mu \mathrm{M}, 48 \mathrm{~h}$ & $\downarrow \sim 38 \%$ colony & $\downarrow \sim 90 \%$ colony & {$[51]$} \\
\hline Colon & LOVO & $10 \mu \mathrm{M}, 48 \mathrm{~h}$ & $\downarrow \sim 10 \%$ colony & Gefitinib & $2 \mu \mathrm{M}, 48 \mathrm{~h}$ & $\downarrow \sim 47 \%$ colony & $\downarrow \sim 90 \%$ colony & {$[51]$} \\
\hline $\mathrm{NSCL} *$ & H1299 & $1 \mu \mathrm{M}, 72 \mathrm{~h}$ & $\sim 7.5 \%$ apoptotic cells & Gefitinib & $1 \mu \mathrm{M}, 72 \mathrm{~h}$ & $\sim 12 \%$ apoptotic cells & $\sim 24 \%$ apoptotic cells & {$[50]$} \\
\hline $\mathrm{NSCL} *$ & H1650 & $1 \mu \mathrm{M}, 72 \mathrm{~h}$ & $\sim 7.5 \%$ apoptotic cells & Gefitinib & $1 \mu \mathrm{M}, 72 \mathrm{~h}$ & $\sim 11 \%$ apoptotic cells & $\sim 25 \%$ apoptotic cells & {$[50]$} \\
\hline
\end{tabular}

Abbreviations: *AML= acute myeloid leukemia; NSCLC= non-small cell lung cancer; [Conversion factor, $228 \mathrm{ng} / \mathrm{ml}=1 \mu \mathrm{M}$ decitabine, $10^{-6} \mathrm{M}=1 \mu \mathrm{M}$ ].

Table 5 In vitro antineoplastic action of decitabine (DAC) in combination with non-toxic agents for the treatment of cancer.

\begin{tabular}{|c|c|c|c|c|c|c|c|c|}
\hline Cancer & Cell Line & $\begin{array}{l}\text { DAC/ Dose } \\
\text { schedule }\end{array}$ & $\begin{array}{c}D A C \\
\text { activity }\end{array}$ & Non-toxic agent & $\begin{array}{l}\text { Agent } \\
\text { conc }\end{array}$ & $\begin{array}{l}\text { Agent } \\
\text { activity }\end{array}$ & $\begin{array}{c}\text { Combination } \\
\text { activity }\end{array}$ & Ref. \\
\hline $\mathrm{AML}^{*}$ & HL-60 & $0.044 \mu \mathrm{M}, 48 \mathrm{~h}$ & $\downarrow 17 \%$ colony & Genistein & $1 \mu \mathrm{M}, 48 \mathrm{~h}$ & $\downarrow 15 \%$ colony & $\downarrow \sim 48 \%$ colony & {$[23]$} \\
\hline $\mathrm{AML}^{*}$ & MOLT-3 & $0.044 \mu \mathrm{M}, 48 \mathrm{~h}$ & $\downarrow 28 \%$ colony & Genistein & $1 \mu \mathrm{M}, 48 \mathrm{~h}$ & $\downarrow 16 \%$ colony & $\downarrow \sim 52 \%$ colony & [23] \\
\hline $\mathrm{AML}^{*}$ & HL-60 & $0.05 \mu \mathrm{M}, 96 \mathrm{~h}$ & $\downarrow 49 \%$ colony & $16-23-D^{*}$ & $10 \mathrm{nM}, 96 \mathrm{~h}$ & $\downarrow 9 \%$ colony & $\downarrow 79 \%$ colony & {$[24]$} \\
\hline $\mathrm{AML}^{*}$ & HL-60 & $0.05 \mu \mathrm{M}, 96 \mathrm{~h}$ & $\downarrow 49 \%$ colony & Vitamin D & $50 \mathrm{nM}, 96 \mathrm{~h}$ & $\downarrow 10 \%$ colony & $\downarrow 78 \%$ colony & {$[24]$} \\
\hline $\mathrm{AML}^{*}$ & HL-60 & $0.05 \mu \mathrm{M}, 48 \mathrm{~h}$ & $\downarrow 38 \%$ colony & Retinoic Acid & $1 \mu \mathrm{M}, 48 \mathrm{~h}$ & $\downarrow 6 \%$ colony & $\downarrow 50 \%$ colony & [52] \\
\hline Colon & DLD-1 & $.05 \mu \mathrm{M}, 120 \mathrm{hr}$ & $\downarrow 28 \%$ colony & Retinoic Acid & $10 \mu \mathrm{M}, 120 \mathrm{hr}$ & $\downarrow 2 \%$ colony & $\downarrow 76 \%$ colony & {$[67]$} \\
\hline
\end{tabular}

Abbreviations: *AML= acute myeloid leukemia; Vitamin D analogues= 16-23-D: 1,25-(OH)2-16-ene-23-yne vitamin D3; [Conversion factor, 228 ng/ml = 1 $\mu \mathrm{M}$ decitabine, $\left.10^{-6} \mathrm{M}=1 \mu \mathrm{M}\right]$.

Table 6 In vivo antineoplastic action of decitabine (DAC) in combination with HDAC inhibitors for the treatment of hematologic malignancies and solid tumors.

\begin{tabular}{|c|c|c|c|c|c|c|c|c|c|}
\hline Cancer & Cell Line & $\begin{array}{l}\text { Mouse } \\
\text { Model }\end{array}$ & DAC/ Dose schedule & $\begin{array}{c}\text { DAC } \\
\text { activity }\end{array}$ & $\begin{array}{c}\text { HDAC } \\
\text { inhibitor }\end{array}$ & HDAC dose & $\begin{array}{c}\text { HDAC } \\
\text { activity }\end{array}$ & $\begin{array}{c}\text { Combination } \\
\text { activity }\end{array}$ & Ref. \\
\hline Leukemia & L1210 & $\mathrm{CD} 2 \mathrm{~F} 1$ & $\begin{array}{l}0.4 \mathrm{mg} / \mathrm{kg} \\
\text { ip: } 2 / \mathrm{d}\end{array}$ & $30.4 \%$ ILS & Phenyl butyrate & $\begin{array}{l}150 \mathrm{mg} / \mathrm{kg} \\
\text { ip: } 2 / \mathrm{d}\end{array}$ & $<1 \%$ ILS & $44.9 \%$ ILS & {$[12]$} \\
\hline Ovarian & SKOv3 & SCID & $\begin{array}{l}5 \mu \mathrm{M}, 48 \mathrm{~h} \\
\text { ex-vivo }\end{array}$ & $\begin{array}{l}\downarrow \sim 43 \% \text { tumor } \\
\text { volume }\end{array}$ & Trichostatin & $\begin{array}{l}0.1 \mu \mathrm{M}, 48 \mathrm{~h} \\
\text { ex-vivo }\end{array}$ & $\begin{array}{l}\downarrow \sim 71 \% \text { tumor } \\
\text { volume }\end{array}$ & $\begin{array}{l}\downarrow \sim 93 \% \text { tumor } \\
\text { volume }\end{array}$ & [39] \\
\hline Ovarian & Hey & Balb-c & $\begin{array}{l}0.8 \mathrm{mg} / \mathrm{kg} \\
\text { ip: 3/week }\end{array}$ & $\sim 25 \%$ ILS & SAHA* & $\begin{array}{l}12.5 \text { mg/kg } \\
\text { ip: } 5 / \text { week }\end{array}$ & $-5 \%$ ILS** & $\sim 40 \%$ ILS & [41] \\
\hline DLDCL* & Ly1 & SCID & $1.5 \mathrm{mg} / \mathrm{kg} \mathrm{d} 1,3, \& 5$ & $\begin{array}{l}\downarrow \sim 18 \% \text { tumor } \\
\text { volume }\end{array}$ & Panobinostat & $\begin{array}{l}15 \mathrm{mg} / \mathrm{kg} \\
\mathrm{d} 1-5\end{array}$ & $\begin{array}{l}\downarrow \sim 30 \% \text { tumor } \\
\text { volume }\end{array}$ & $\begin{array}{l}\downarrow \sim 88 \% \text { tumor } \\
\text { volume }\end{array}$ & {$[28]$} \\
\hline
\end{tabular}

Abbreviations: *DLBCL= diffuse large B-cell lymphoma; SAHA= suberoylanilide hydroxamic acid; ** toxicity.

Table 7 In vivo antineoplastic action of decitabine (DAC) in combination with different agents for the treatment of hematologic malignancies and solid tumors.

\begin{tabular}{|c|c|c|c|c|c|c|c|c|c|}
\hline Cancer & Cell Line & Mouse Model & $\begin{array}{l}\text { DAC/ Dose } \\
\text { schedule }\end{array}$ & $\begin{array}{c}D A C \\
\text { activity }\end{array}$ & Agent & Dose schedule & $\begin{array}{l}\text { Drug } \\
\text { activity }\end{array}$ & $\begin{array}{c}\text { Combination } \\
\text { activity }\end{array}$ & Ref. \\
\hline AML & $\begin{array}{l}\text { U937 } \\
\text { [SubQ] }\end{array}$ & NOD/SCID & $\begin{array}{l}0.5 \mathrm{mg} / \mathrm{kg} \\
5 \text { days }\end{array}$ & $\begin{array}{l}\downarrow \sim 7 \% \text { tumor } \\
\text { volume }\end{array}$ & Idarubicin & $\begin{array}{l}0.5 \mathrm{mg} / \mathrm{kg} \\
3 \text { days }\end{array}$ & $\begin{array}{l}\downarrow \sim 20 \% \text { tumor } \\
\text { volume }\end{array}$ & $\begin{array}{l}\downarrow \sim 47 \% \text { tumor } \\
\text { volume }^{\star \star}\end{array}$ & {$[68]$} \\
\hline Leukemia & L1210 & $\mathrm{CD} 2 \mathrm{~F} 1$ & $\begin{array}{l}6.25 \mathrm{mg} / \mathrm{kg} \\
15 \mathrm{~h} \text { i.v. infus }\end{array}$ & $\sim 80 \%$ ILS & 3- DU* & $\begin{array}{l}150 \mathrm{mg} / \mathrm{kg} \\
15 \mathrm{~h} \text { i.v. infus }\end{array}$ & $\sim 30 \%$ ILS & $>70 \%$ cured mice & [16] \\
\hline Leukemia & L1210 & BALB-c & $\begin{array}{l}2 \mathrm{mg} / \mathrm{kg} \\
15 \mathrm{~h} \text { i.v. infus }\end{array}$ & $\sim 40 \%$ ILS & Zebularine & $\begin{array}{l}20 \mathrm{mg} / \mathrm{kg} \\
15 \mathrm{~h} \text { i.v. infus }\end{array}$ & $\begin{array}{l}\text { same as } \\
\text { control }\end{array}$ & 90\%ILS & {$[44]$} \\
\hline Leukemia & L1210 & $\mathrm{CD} 2 \mathrm{~F} 1$ & $\begin{array}{l}1 \mathrm{mg} / \mathrm{kg} \\
\text { ip: } 5 \times 4 \mathrm{~h}\end{array}$ & $156 \%$ ILS & Zebularine & $\begin{array}{l}20 \mathrm{mg} / \mathrm{kg} \\
1 \text { injection }\end{array}$ & $7 \%$ ILS & $244 \%$ ILS & [9] \\
\hline Leukemia & L1210 & $\mathrm{CD} 2 \mathrm{~F} 1$ & $\begin{array}{l}2 \mathrm{mg} / \mathrm{kg} \\
12 \mathrm{~h} \text { i.v. infus }\end{array}$ & $76.6 \%$ ILS & DZNep* & $\begin{array}{l}2.5 \mathrm{mg} / \mathrm{kg} \\
\text { i.p at } 1 \mathrm{~h} \& 6 \mathrm{~h}\end{array}$ & $22.1 \%$ ILS & $106.5 \%$ ILS & {$[47]$} \\
\hline Leukemia & L1210 & $\mathrm{CD} 2 \mathrm{~F} 1$ & $\begin{array}{l}2 \mathrm{mg} / \mathrm{kg} \\
8 \mathrm{~h} \text { i.v. infus }\end{array}$ & $73 \%$ ILS & Genistein & $\begin{array}{l}0.5 \% \text { enriched } \\
\text { diet }\end{array}$ & $8 \%$ ILS & $94 \%$ ILS & [23] \\
\hline Colorectal & SW48 & BALB-C & $\begin{array}{l}3 \mathrm{mg} / \mathrm{kg} \times 3 \\
\text { day }-6\end{array}$ & $\begin{array}{l}\downarrow \sim 8 \% \text { tumor } \\
\text { volume }\end{array}$ & 5- $\mathrm{FU}$ * & $\begin{array}{c}20 \mathrm{mg} / \mathrm{kg} \\
\text { days } 0-4\end{array}$ & $\begin{array}{l}\downarrow \sim 32 \% \text { tumor } \\
\text { volume }\end{array}$ & $\begin{array}{l}\downarrow \sim 44 \% \text { tumor } \\
\text { volume }\end{array}$ & {$[69]$} \\
\hline
\end{tabular}

Abbreviations: *5-FU= 5-fluorouracil; DZNep= 3-deazaneplanocin A; 3-DU= 3-deazauridine; ILS= increase in lifespan; ** sequential treatment. 


\begin{tabular}{|c|c|c|c|c|c|c|c|c|c|}
\hline $\begin{array}{l}\text { Study } \\
\text { Phase }\end{array}$ & Indication* & Patients & Age & $\begin{array}{c}\text { Dose schedule } \\
\text { DAC }\end{array}$ & $\begin{array}{l}\text { Second } \\
\text { drug }\end{array}$ & $\begin{array}{l}\text { Dose schedule of } \\
\text { drug }\end{array}$ & Results** & Comments & Ref. \\
\hline I & AML & 16 & $\begin{array}{l}50 \%>60 y r s \\
50 \%>18 y r s\end{array}$ & $\begin{array}{l}20 \mathrm{mg} / \mathrm{m} 2 \mathrm{IV} \\
\text { daily on days } 1 \text { - } \\
5 \text { repeated Q28 } \\
\text { days }\end{array}$ & $\begin{array}{l}\text { Midostaurin } \\
\text { (sequential) }\end{array}$ & $\begin{array}{l}50 \text { mg bid days } \\
8 \text { - } 21 \text { of each } \\
\text { cycle }\end{array}$ & $\begin{array}{l}57 \% \text { SD } \\
25 \% \text { CHR }\end{array}$ & - & [49] \\
\hline$|/ I|$ & $\begin{array}{l}\text { MDS and } \\
\text { AML }\end{array}$ & $\begin{array}{l}54 \\
(48 \mathrm{AML} \\
6 \mathrm{MDS})\end{array}$ & $>2 \mathrm{yrs}$ & $\begin{array}{l}15 \mathrm{mg} / \mathrm{m} 2 \text { by IV } \\
\text { daily for } 10 \text { days }\end{array}$ & Valproic Acid & $\begin{array}{l}50 \mathrm{mg} / \mathrm{kg} \text { daily } \\
\text { dose for } 10 \text { days }\end{array}$ & $\begin{array}{l}22 \% \text { OR, } 19 \% \text { CR } \\
3 \% \text { CRp }\end{array}$ & $\begin{array}{l}\text { Overall Survival: } \\
15.3 \text { months }\end{array}$ & [53] \\
\hline 1 & AML & 25 & $70 \mathrm{yrs}$ & $\begin{array}{l}20 \mathrm{mg} / \mathrm{m} 2 \text { daily } \\
\text { during } 1 \mathrm{hr} \text { for } \\
10 \text { consecutive } \\
\text { days }\end{array}$ & Valproic Acid & $20 \mathrm{mg} / \mathrm{kg} /$ daily & $\begin{array}{l}44 \% \text { RR } \\
52 \%(11) \\
\text { responded: } \\
4 \text { CR, } 4 \text { iCR, } 3 \text { PR }\end{array}$ & $\begin{array}{l}\text { Dose limited } \\
\text { by neurologic } \\
\text { adverse effects }\end{array}$ & {$[70]$} \\
\hline ॥ & $\begin{array}{l}\text { MDS and } \\
\text { AML }\end{array}$ & $\begin{array}{l}149 \\
\text { (87 MDS, } \\
62 \mathrm{AML})\end{array}$ & $69 \mathrm{yrs}$ & $\begin{array}{l}20 \text { mg/m2 daily } \\
\text { for } \\
5 \text { days repeated } \\
\text { every } 4 \text { - } 6 \\
\text { weeks }\end{array}$ & Valproic Acid & $\begin{array}{l}50 \mathrm{mg} / \mathrm{kg} \text { daily } \\
\text { for } \\
7 \text { days repeated } \\
\text { every } 4 \text { - } 6 \text { weeks }\end{array}$ & $\begin{array}{l}34 \% \text { CR } \\
55 \% \text { OR }\end{array}$ & $\begin{array}{l}\text { Median Survival: } \\
11.9 \text { months } \\
\text { Estimated 2-yr } \\
\text { survival rate: } 27 \%\end{array}$ & [71] \\
\hline 1 & $\begin{array}{l}\text { Ovarian } \\
(\mathrm{EOC})\end{array}$ & 9 & $62.5 \mathrm{yrs}$ & $\begin{array}{l}10 \mathrm{mg} / \mathrm{m} 2 \text { or } 20 \\
\mathrm{mg} / \mathrm{m} 2 \text { IV daily } \\
\text { for } 5 \text { days }\end{array}$ & Carboplatin & $\begin{array}{l}\text { I.V bolus on day } \\
8 \text { of a } 28 \text {-day } \\
\text { cycle }\end{array}$ & $\begin{array}{l}10 \% \text { CR } \\
60 \% \text { SD }\end{array}$ & & {$[72]$} \\
\hline 1 & NSCLC & 8 & $\begin{array}{l}>\text { or equal } \\
18 \text { yrs }\end{array}$ & $\begin{array}{l}5-15 \mathrm{mg} / \mathrm{m} \text { for } \\
\text { 10days }\end{array}$ & Valproic Acid & $\begin{array}{l}10-20 \mathrm{mg} / \mathrm{kg} / \\
\text { day PO on days } \\
5-21 \text { of a } 28 \text {-day } \\
\text { cycle. }\end{array}$ & $\begin{array}{l}\text { Limited by } \\
\text { unacceptable } \\
\text { neurological } \\
\text { toxicity at a } \\
\text { relatively low } \\
\text { dosage }\end{array}$ & & {$[54]$} \\
\hline 1 & $\begin{array}{l}\text { Advanced } \\
\text { solid } \\
\text { tumors or } \\
\text { NHLs }\end{array}$ & 43 & - & $\begin{array}{l}10 \mathrm{mg} / \mathrm{m} 2 \text { daily } \\
\text { IV. on days } 1 \text { - } 5\end{array}$ & Vorinostat & $\begin{array}{l}200 \text { mg, } 2 \text { daily } \\
\text { either } \\
\text { sequential. }\end{array}$ & $\begin{array}{l}\text { Disease } \\
\text { stabilization for } \\
\text { four or more } \\
\text { cycles was } \\
\text { observed in } \\
\text { about 30\% of } \\
\text { patients }\end{array}$ & & [55] \\
\hline
\end{tabular}

Abbreviations: * AML= acute myeloid leukemia; MDS= myelodysplastic syndrome; EOC=epithelial ovarian carcinoma; NSCLC= non-small-cell lung cancer; $\mathrm{NHL}=$ non-Hogkin lymphoma; **SD: stable disease; RR: response rate; CR: complete response; CRp: CR with incomplete recovery of platelet counts; PR: partial response; CHR: clinically high risk.

effective and tolerable for inhibiting tumor growth in a biologic model of the lymphoma (Table 6).

\section{Valproic acid (VPA)}

VPA, a short chain fatty acid, has been used as an antiepileptic and mood stabilizer [29]. It also is considered to be a weak inhibitor of HDAC. The effects of VPA, alone and in combination with DAC, were examined with respect to the growth and survival of the human leukemia cells MOLT4 and $\mathrm{HL}-60$ (Table 1). The sequential administration of DAC and VPA resulted in a $90 \%$ reduction of viable cells, which was greater than either agent alone. This combination has a potentially synergistic activity [30].

\section{Depsipeptide (Depsi)}

In 1998, Depsi, a bicyclic peptide, was demonstrated to be an HDAC inhibitor [31]. Depsi activity has been reported in numerous in vitro studies, that used it in combination with DAC for hematologic malignancies or solid tumors. A report using in vitro assays showed that the combination of DAC and depsi produced a greater antineoplastic effect against Raji lymphoma cells than either agent alone. In a colony assay, the loss of clonogenicity produced by DAC or depsi as single agents was about $28 \%$ and $27 \%$, respectively. The combination of these two drugs increased the loss of clonogenicity synergistically by $81 \%$ [32] (Table 1). In a different study on AML, the activity of depsi was enhanced by DAC. The combination of these two agents resulted in enhanced histone acetylation and cytotoxicity [33]. Two other studies also investigated the capacity of DAC and depsi to inhibit colony formation in breast carcinoma cell lines $[34,35]$. Both drugs were potent agents for inhibiting colony formation, and their combination produced a synergistic antineoplastic effect (Table 2).

\section{Phenylbutyrate $(P B)$}

$\mathrm{PB}$, an inhibitor of HDAC, increases histone acetylation and leads to chromatin with a more open and accessible structure. The in vitro treatment of DAC and PB in combination produced a greater inhibition of growth, DNA synthesis, and greater reduction of colony formation in L1210 and human HL-60 leukemic cells as compared to either drug alone. This combination also enhanced the antineoplastic activity in mice with L1210 leukemia [12]. The effects of DAC and PB -alone or in combination- on the loss of clonogenicity for the lung cancer cell lines A549 and Calu- 6 are shown in Table 2. A significant synergistic antitumor effect was observed in the Calu- 6 cells clonogenic assay. DAC produced a $25 \%$ loss of clonogenicity, whereas PB produced only $10 \%$. The combination of the drugs increased the loss of clonogenicity to about 75\% [36].

\section{Trichostatin A (TSA)}

TSA is an antifungal antibiotic that selectively inhibits class I and II HDACs [37]. TSA is a model HDAC inhibitor that is not used in clinical therapy. The combination of DAC and TSA was synergistic with respect to the cell kill of the MDA-MB -231 breast carcinoma (Table 2). The sequential treatment 
of the malignant cell line, first with DAC and subsequently with TSA, resulted in a more robust re-expression of the methylated tumor suppressor genes, such as the retinoic acid receptor and the estrogen receptor alpha gene [38]. In an in vivo study, the combination of TSA and DAC was tested by evaluating the tumorigenicity of pretreated SKOV3 ovarian cancer cells in mouse xenograft models. This study found that the combination suppressed tumor formation to a greater extent than when either agent was used alone. The combination treatment lead to an almost 93\% decrease in tumor volume, which was statistically significant (Table 6) [39].

\section{Vorinostat (SAHA)}

Vorinostat (suberoylanilide hydroxamic acid [SAHA]), a hydroxamic acid derivative, was one of the first HDACs to be commercially available and used in oncology [40]. The combination of DAC and SAHA produced a synergistic inhibition of Hey and SKOV3 ovarian cell growth by apoptosis and cell cycle arrest (Table 2), and inhibited the growth of Hey xenografts (Table 6) [41]. This combination also enhanced the antiproliferative effect on pancreatic cells as shown in Table 2 [42].

\section{Inhibitor of DNA methylation and cytidine deaminase}

\section{Zebularine (Zeb)}

Zeb is a stable nucleoside analog that is suitable for oral administration. It was originally identified as a cytidine deaminase inhibitor, but this compound also inhibits DNA methylation [43]. The effect of Zeb on the antineoplastic action of DAC in V5 cells transduced with the CR deaminase gene was investigated using an in vitro colony assay. For these cells, the loss of the clonogenicity of DAC plus Zeb was greater than DAC or Zeb alone [44]. In another study, this combination produced a greater inhibition of the growth of murine L1210 lymphoid leukemia cells, and a greater reduction in the colony formation of L1210 and human HL60 myeloid leukemia cells, than either agent alone. In the mouse model of L1210 leukemia (Table 7; schedule A), the combination produced a significantly greater ILS (244\%) as compared to DAC alone (156\% ILS) or Zeb alone ( $7 \%$ ILS) [9]. Other schedules for this combination were tested and were as promising with minimal toxicity (less than a 3\% loss in body weight) [9]. Zeb also was tested in combination with respect to human bladder cancer cells [45].

\section{Inhibitor of DAC resistant cells}

\section{3- Deazauridine (3- DU)}

3-DU is a CTP synthetase inhibitor that is effective against leukemic cells deficient in deoxycytidine kinase (DCK). In one study, 3-DU was used in combination with DAC, which resulted in an enhancement of the in vitro antineoplastic action of DAC on human leukemic cells. Using an optimized dose-schedule, this study showed that this combination could cure some mice bearing L1210 leukemia, even in the presence of a subpopulation of drug resistant (L1210/ ARA-C) leukemia cells lacking DCK. Interestingly, the combination maintained a high percentage of cured mice (70\%). The results of the administration of a $15 \mathrm{~h}$ infusion of DAC alone or in combination with 3-DU are shown in Table 7 [17].

\section{Inhibitor of histone methylation}

\section{3- Deazaneplanocin (DZNep)}

DZNep, an inhibitor of methionine metabolism, can reactivate genes silenced by lysine 27 histone 3 (H3K27) me3 by inhibiting EZH2 [46]. A study investigating the antileukemic action of the inhibitors of DNA methylation and histone methylation assessed human HL-60 and murine L1210 leukemia cells exposed in vitro to DAC combined with DZNep. This study showed a synergistic loss of clonogenicity in colony assay as compared to each agent alone (Table 3) [47]. This positive chemotherapeutic interaction was observed in mice with L1210 leukemia (Table 7). The colony assay of leukemic cells after a sequential treatment with DAC and DZNep showed a synergistic reduction of cell survival. An almost $90 \%$ reduction in the survival of $\mathrm{HL}-60$ and $\mathrm{AML}-3$ cell lines was observed with the double combination (Table 1). A triple combination also was tested using DAC, DZNep and TSA which induced a remarkable synergistic antineoplastic effect in human AML cells that was demonstrated by an in vitro colony assay. The reduction in colony formation of HL-60 leukemic cells by DAC; DZNep; TSA; DAC + DZNepp; DAC + TSA; DZNep + TSA; and DAC + DZNep + TSA waS $22,35,24,64,45,90$ and $<1 \%$, respectively. [46]

\section{Tyrosine kinase Inhibitors}

\section{Midostaurin (MS)}

MS is a potent inhibitor of Fms-like tyrosine kinase 3 (FLT3), and other molecular targets thought to be important to the pathogenesis of AML [48]. Compared to treatment with either agent alone, co-treatment or sequential treatment with DAC and MS synergistically induce greater apoptosis of cultured FLT3-ITD expressing AML cells (Table 4) [49].

\section{Gefitinib (GEF)}

GEF is an inhibitor of epidermal growth factor receptor (EGFR) that could be used potentially to treat solid tumors, such as non-small lung cancer and colon cancer. Compared to either DAC or gefitinib treatment alone, a significant additional increase in apoptosis was observed in the H1650 lung carcinoma cells treated with a combination of DAC and gefitinib for $72 \mathrm{hrs}$ as determined by Annexin $\mathrm{V}$ staining [50]. The synergistic antineoplastic effect of DAC and gefitinib was observed in SW1116 and LOVO colon cancer cells as evaluated by clonogenic assay [51]. The combined activity is presented in Table 4. One advantage of using tyrosine kinase inhibitors in combination with DAC is that they do not produce any hematopoietic toxicity.

\section{Nontoxic agents}

\section{Genistein}

The antileukemic activity of a treatment that uses DAC combined with genistein (non-toxic soybean isoflavone) has been investigated. The chemotherapeutic potential of co-treatment was assessed in leukemia cells using an in vitro clonogenic assay (Table 5), and its in vivo antineoplastic activity was assessed in a mouse model of L1210 leukemia (Table 7) [23]. The results of this study suggested that the combined treatment of DAC and genistein has a promising antineoplastic activity that was greater than either agent alone. 


\section{Vitamin D}

The antineoplastic action of DAC was investigated in combination with vitamin D analogs in HL-60 and NB-4 myeloid leukemia cells. The combination of DAC with either of the vitamin $D$ analogs produced a greater growth inhibition and induction of differentiation than either agent alone. The loss of clonogenicity produced by DAC and 1,25-(OH)2-16-ene-23-yne vitamin D3 in HL-60 leukemia cells after $96 \mathrm{~h}$ exposure produced an almost $80 \%$ loss of clonogenicity as opposed to an almost $10 \%$ loss with the vitamin D analog alone or a 50\% loss with DAC alone (Table 5) [24].

\section{Retinoic acid (ATRA)}

Retinoic acid, a metabolite of vitamin A, is an interesting agent to use in combination with DAC. Using a colony assay, a synergistic antineoplastic interaction was observed between ATRA and DAC on the DLD-1 colon cells. With a $120 \mathrm{~h}$ exposure, DAC alone produced a $28 \%$ loss of clonogenicity, whereas ATRA alone produced only a $2 \%$ loss of clonogenicity. The combination resulted in a $76 \%$ loss of clonogenicity - a significant synergistic interaction [67]. In another study, this combination was assessed in HL-60 leukemia cells where a synergistic effect also was observed (Table 5) [52].

\section{Combinations in clinical studies}

Clinical studies that evaluate combination regimens with positive outcomes need to be analyzed, so practitioners can incorporate them into their clinical practices. A phase I trial was performed in patients with $A M L$ where the maximum tolerated dose (MTD) and schedule of administration of the combination was identified as DAC followed by the tyrosine kinase inhibitor midostaurin (MS). Based on an intent-to-treat analysis, $57 \%$ of the patients achieved stable disease (SD) or better while enrolled in the trial; and $25 \%$ had a complete hematologic response (CHR). The results of this clinical study suggested that DAC with sequential MS can be given safely to most patients in an outpatient setting. However, it also should be noted that in one cohort, the concomitant administration of DAC and MS resulted in a fatal dose limiting pulmonary toxicity in two of three patients [49].

A number of phase I/II clinical studies have looked at the combination of the HDAC inhibitor valproic acid (VPA) with DAC in the treatment of MDS and AML. Low-dose DAC with VPA leads to a somewhat improved outcome in the treatment of elderly patients with AML. Neurotoxicity was more common when VPA was added to the DAC versus DAC alone. Clinical improvements, including complete responses, were noted in patients treated with or without VPA [53]. This combination was also investigated in advanced stage IV non-small-cell lung carcinoma (NSCLC) cancer; although the combination was effective in reactivating the hypermethylated genes, it was limited by unacceptable neurological deficits at a relatively low dosage [54].

In a phase I study, the safety and efficacy of the HDAC inhibitor vorinostat was evaluated. The combination of DAC and vorinostat was tested in patients with advanced solid tumors or non-Hodgkin's lymphomas. The combination showed activity with prolonged disease stabilization in different tumor types. Dose limiting toxicities during the first cycle consisted of myelosuppression and gastrointestinal symptoms. These symptoms occurred in $29 \%$ of the patients that could be evaluated for toxicity [55]. The main outcomes of these studies are presented in Table 8.

\section{Discussion}

The epigenome is another potential target for investigation in anticancer therapy. Taking into consideration the diversity of cancer cells, epigenetics is one of the most important components in the development of personalized oncology. The hypermethylation of cytosines in CpG dinucleotides in the promoter regions of tumor suppressor genes is an important mechanism of gene silencing. The deacetylation of histones in nucleosomes represents a second key epigenetic mechanism of gene silencing that also can contribute to tumor formation and progression [56]. A pharmacodynamic and pharmacokinetic analysis of DAC from both preclinical and clinical data can provide insight towards the optimization of cancer treatment using this interesting epigenetic agent $[57,58]$. It is important to select a very effective dose-schedule for DAC before proceeding to studies on combination chemotherapy. Current studies are evaluating the use of DAC in combination therapy as a possibility of inducing a remission in patients that do not respond to standard therapy. The clinical investigation of drug combinations requires single drugs to be proven to be efficient, preclinical data to confirm the synergism of both drugs, the combination of the two drugs to be evaluated for maximal tolerated dose and the efficacy of a drug combination [59]. In this current review, we looked at the combination therapy of DAC in preclinical and clinical studies.

Amongst different agents, HDAC inhibitors have been given a priority for clinical studies in combination with DAC. In addition, accumulating evidence suggests that the simultaneous hypomethylation of DNA promoters and the acetylation of histones can produce the synergistic re-expression of silenced genes [60]. Other hypothetical explanations for this response include alterations in differentiation, changes in apoptosis and an induction of beneficial immune response [9]. The data supporting any particular mechanism are limited. A number of different compounds inhibit HDAC, including the short chain fatty acids phenyl butyrate and valproic acid (VPA), the benzamides SNDX-275, the cyclic peptide romidepsin, and the hydroxamic acids vorinostat and trichostatin A. As a class, these agents are limited by toxicity due to myelosuppression, fatigue, and gastrointestinal symptoms [61]. The sequence of drug administration is important. HDAC inhibitors can block the progression of G1 cells into the $S$ phase. This action can interfere with the antineoplastic action of DAC, which is an S phase specific drug. The best schedule is sequential: first DAC followed by the HDAC inhibitor. This rationale also is applicable to EZH2 inhibitors, which can also block cell cycle progression [46]. Other DNMT agents can be used in combination with DAC. Zebularine exhibits DNA demethylation activity with 
reduced potency and toxicity in comparison to DAC [15]. Zebularine also inhibits CR deaminase, the enzyme that inactivates DAC. Combination chemotherapy using agents that act as signal transduction modulators and non-toxic agents with fewer side effects are interesting agents for investigation.

Major gaps exist in the ability to develop rational hypotheses about the toxicity profile of DAC when used in combination during clinical trials for cancer. The gap represented by the limited data for any agent regarding its mechanism of toxicity considerably restricts the ability to predict the safety of combining different agents with DAC. The major side effect of DAC is hematopoietic toxicity. If the agent to be used in combination also exhibits hematopoietic toxicity, a conservative dose-schedule should be used for the second agent in the initial studies. Modification of the dose-schedule of the second agent can be done according to the extent of its hematopoietic toxicity. The use of epigenetic drugs in combination with DAC showed limited success in high risk MDS/AML, but not so with patients previously treated with several lines of therapy and solid tumors [6]. It is still not clear which is the most effective HDAC inhibitor to use with DAC, or the optimal sequence and schedule. Moreover, it remains to be clarified whether the mechanisms of these agents are solely epigenetic in nature, or whether the inhibitors also have some cytotoxic activity. In this context, data are emerging suggesting that an interference with the DNMT function can disrupt DNA repair complexes, which leads to DNA damage [50]. Further investigation into the induction of DNA damage, apoptosis, differentiation and immune modulation are necessary to determine which, if any, of these mechanisms are responsible for clinical efficacy. Another challenge is the dosing schedules for DAC, since uncertainty exists as to the best dose to use clinically, and several studies have tried to optimize a schedule [57] [58]. This uncertainty continues because of the dual mechanism of action of DAC, its hypomethylation at low doses and cytotoxicity at high doses [62]. Clinical studies represent only a small number of patients, which renders it impossible to draw any definitive conclusions regarding the superiority of any of the dose levels studies. Also, it is more challenging to carry out comparative studies, since it is difficult to establish whether stable disease is due to the combination of the two agents and what the expected outcomes would be if each agent was used alone.

This literature review contains comments about the potential use of DAC in combination therapy. Although the review covers a wide range of agents from different drug classes, it is limited in the sense that not all agents that have been used in combination are mentioned here, and not all trials are included. Only a limited number of trials involving combination therapy have been fully reported. Some of the early phase trials of combination therapy have been presented only in abstract form. The review has summarized the preclinical studies and the main outcomes of a few selected clinical trials.

The full chemotherapeutic potential of DAC to treat cancer merits clinical investigation and can only be determined by further assessment of patients with cancer to determine the optimal combination. Combination therapy with the aforementioned agents is an active area of study based on compelling preclinical data. In future clinical trials on DAC in combination with other agents in patients with cancer, it is important to compare the clinical responses of the combination with DAC used as a single agent [7, $63,64]$. The superiority of combination therapy over the use of DAC as a single agent has yet to be proven, and larger randomized trials comparing the two therapies and different dosing regimens are required to ultimately establish whether clinical experience will correlate with preclinical predictions of synergy for these agents. Numerous issues must be resolved for DNMT inhibitors to achieve a broad use in cancer treatment. In particular, drug delivery, optimal dose, appropriate clinical testing, resistance and sensitivity need to be explored further.

\section{Conflict of interest}

The authors declare no conflict of interest.

\section{References}

[1] Momparler RL. Cancer epigenetics. Oncogene. 2003; 22(42):64796483.

[2] Ballestar E, Esteller M. Epigenetic gene regulation in cancer. Adv Genet. 2008; 61:247-267.

[3] Sharma S, Kelly TK, Jones PA. Epigenetics in cancer. Carcinogenesis. 2010; 31(1):27-36

[4] Jüttermann R, Li E, Jaenisch R. Toxicity of 5-aza-2'-deoxycytidine to mammalian cells is mediated primarily by covalent trapping of DNA methyltransferase rather than DNA demethylation. Proc Natl Acad Sci USA. 1994; 91(25):11797-11801.

[5] Jabbour E, Issa JP, Garcia-Manero G, Kantarjian H. Evolution of decitabine development. Cancer. 2008; 112(11):2341-2351.

[6] Kantarjian H, Issa JP, Rosenfeld CS, Bennett JM, Albitar M, et al. Decitabine improves patient outcomes in myelodysplastic syndromes: results of a phase III randomized study. Cancer. 2006; 106(8):1794-1803.

[7] Issa JP, Garcia-Manero G, Giles FJ, Mannari R, Thomas D, et al. Phase 1 study of low-dose prolonged exposure schedules of the hypomethylating agent 5-aza-2'-deoxycytidine (decitabine) in hematopoietic malignancies. Blood. 2004; 103(5):1635-1640.

[8] Momparler RL, Bouffard DY, Momparler LF, Dionne J, Bélanger K, et al. Pilot phase I-II study on 5-aza-2'-deoxycytidine (Decitabine) in patients with metastatic lung cancer. Anti-cancer Drugs. 1997; 8:358368.

[9] Lemaire M, Momparler LF, Bernstein ML, Marquez VE, Momparler RL. Enhancement of antineoplastic action of 5-aza-2-deoxycytidine by zebularine on L1210 leukemia. Anti-cancer Drugs. 2005; 16(3):301308.

[10] Jones PA. DNA methylation and cancer. Oncogene. 2002; 21(35):53585360.

[11] Esteller M. Epigenetics in cancer. New EngIJ Med. 2008; 358(11):11481159.

[12] Lemaire M, Momparler LF, Farinha NJ, Bernstein M, Momparler RL. Enhancement of antineoplastic action of 5-aza-2'-deoxycytidine by phenylbutyrate on L1210 leukemic cells. Leukemia Lymphoma. 2004; 45(1):147-154.

[13] Marks PA, Dokmanovic M. Histone deacetylase inhibitors: discovery and development as anticancer agents. Expert Opin Investig Drugs. 2005; 14(12):1497-1511

[14] Yoo CB, Cheng JC, Jones PA. Zebularine: a new drug for epigenetic therapy. Biochem Soc Trans. 2004; 32(Pt6):910-912.

[15] Zhou L, Cheng X, Connolly BA, Dickman MJ, Hurd PJ, et al. Zebularine: a novel DNA methylation inhibitor that forms a covalent complex with DNA methyltransferases. J Mol Biol. 2002; 321(4):591-599.

[16] Raynal NJ, Momparler LF, Rivard GE, Momparler RL. 3-Deazauridine enhances the antileukemic action of 5-aza-2-deoxycytidine and targets drug-resistance due to deficiency in deoxycytidine kinase. Leuk Res. 2011; 35(1):110-118.

[17] Momparler RL, Veselý J, Momparler LF, Rivard GE. Synergistic action of 5-aza-2'-deoxycytidine and 3-deazauridine on L1210 leukemic cells and EMT6 tumor cells. Cancer Res. 1979; 39(10):3822-3827.

[18] Viré E, Brenner C, Deplus R, Blanchon L, Fraga M, et al. The Polycomb group protein EZH2 directly controls DNA methylation. Nature. 2006; 439(7078):871-874.

[19] Wheeler DL, Dunn EF, Harari PM. Understanding resistance to EGFR inhibitors-impact on future treatment strategies. Nat Rev Clin Oncol. 2010; 7(9):493-507.

[20] Schwartz PA, Kuzmic P, Solowiej J, Bergqvist S, Bolanos B, et al. Covalent EGFR inhibitor analysis reveals importance of reversible interactions to potency and mechanisms of drug resistance. Proc Natl Acad Sci USA. 2014; 111(1):173-178. 
[21] Gudas LJ. Retinoids, retinoid-responsive genes, cell differentiation, and cancer. Cell Growth Differ. 1992; 3(9):655-662.

[22] Huang ME, Ye YC, Chen SR, Chai JR, Lu JX, et al. Use of all-trans retinoic acid in the treatment of acute promyelocytic leukemia. Blood. 1988; 72(2):567-572.

[23] Raynal NJ, Charbonneau M, Momparler LF, Momparler RL. Synergistic effect of 5-Aza-2'-deoxycytidine and genistein in combination against leukemia. Oncol Res. 2008; 17(5):223-230.

[24] Doré BT, Chomienne C, Momparler RL. Effect of 5-aza-2'deoxycytidine and vitamin D3 analogs on growth and differentiation of human myeloid leukemic cells. Cancer Chemother Pharmacol. 1998; 41(4):275-280.

[25] Valeriote F, Lin HS. Synergistic interaction of anticancer agents: a cellular perspective. Cancer Chemother Rep. 1975; 59(5):895-900.

[26] George P, Bali P, Annavarapu S, Scuto A, Fiskus W, et al. Combination of the histone deacetylase inhibitor LBH589 and the hsp90 inhibitor 17-AAG is highly active against human CML-BC cells and AML cells with activating mutation of FLT-3. Blood. 2005; 105(4):1768-1776.

[27] Fiskus W, Buckley K, Rao R, Mandawat A, Yang Y, et al. Panobinostat treatment depletes EZH2 and DNMT1 levels and enhances decitabine mediated de-repression of JunB and loss of survival of human acute leukemia cells. Cancer Biol Ther. 2009; 8(10):939-950.

[28] Kalac M, Scotto L, Marchi E, Amengual J, Seshan VE, et al. HDAC inhibitors and decitabine are highly synergistic and associated with unique gene-expression and epigenetic profiles in models of DLBCL. Blood. 2011; 118(20):5506-5516.

[29] Detich N, Bovenzi V, Szyf M. Valproateinduces replication-independent active DNA demethylation. J Biol Chem. 2003; 278(30):27586-27592.

[30] Yang H, Hoshino K, Sanchez-Gonzalez B, Kantarjian H, Garcia-Manero G. Antileukemia activity of the combination of 5-aza-2'-deoxycytidine with valproic acid. Leuk Res. 2005; 29(7):739-748.

[31] Nakajima H, Kim YB, Terano H, Yoshida M, Horinouchi S. FR901228, a potent antitumor antibiotic, is a novel histone deacetylase inhibitor. Exp Cell Res. 1998; 241(1):126-133.

[32] Shaker S, Bernstein M, Momparler RL. Antineoplastic action of 5-aza2'-deoxycytidine (Dacogen) and depsipeptide on Raji lymphoma cells. Oncol Rep. 2004; 11(6):1253-1256.

[33] Shaker S, Bernstein M, Momparler LF, Momparler RL. Preclinical evaluation of antineoplastic activity of inhibitors of DNA methylation (5-aza-2'-deoxycytidine) and histone deacetylation (trichostatin A, depsipeptide) in combination against myeloid leukemic cells. Leuk Res. 2003; 27(5):437-444.

[34] Primeau M, Gagnon J, Momparler RL. Synergistic antineoplastic action of DNA methylation inhibitor 5-AZA-2'-deoxycytidine and histone deacetylase inhibitor depsipeptide on human breast carcinoma cells. Int J Cancer. 2003; 103(2): 177-184

[35] Gagnon J, Shaker S, Primeau M, Hurtubise A, Momparler RL. Interaction of 5-aza-2'-deoxycytidine and depsipeptide on antineoplastic activity and activation of 14-3-3sigma, E-cadherin and tissue inhibitor of metalloproteinase 3 expression in human breast carcinoma cells. Anti-cancer Drugs. 2003; 14:193-202.

[36] Boivin AJ, Momparler LF, Hurtubise A, Momparler RL. Antineoplastic action of 5-aza-2'-deoxycytidine and phenylbutyrate on human lung carcinoma cells. Anti-cancer. 2002; 13(8): 869-874.

[37] Vanhaecke T, Papeleu P, Elaut G, Rogiers V. Trichostatin A-like hydroxamate histone deacetylase inhibitors as therapeutic agents: toxicological point of view. Curr Med Chem. 2004; 11(12):16291643.

[38] Bovenzi V, Momparler RL. Antineoplastic action of 5-aza-2'deoxycytidine and histone deacetylase inhibitor and their effect on the expression of retinoic acid receptor beta and estrogen receptor alpha genes in breast carcinoma cells. Cancer Chemother Pharmacol. 2001; 48(1):71-76. (Pubmed)

[39] Meng F, Sun G, Zhong M, Yu Y, Brewer MA. Inhibition of DNA methyltransferases, histone deacetylases and lysine-specific demethylase- 1 suppresses the tumorigenicity of the ovarian cancer ascites cell line SKOV3. Int J Oncol. 2013; 43(2):495-502.

[40] Siegel D, Hussein M, Belani C, Robert F, Galanis E, et al. Vorinostat in solid and hematologic malignancies. J Hematol Oncol. 2009; 2:3142

[41] Chen MY, Liao WS, Lu Z, Bornmann WG, Hennessey V, Washington MN, et al. Decitabine and suberoylanilide hydroxamic acid (SAHA) inhibit growth of ovarian cancer cell lines and xenografts while inducing expression of imprinted tumor suppressor genes, apoptosis, G2/M arrest, and autophagy. Cancer. 2011: 117(19):4424-4438.

[42] Kumagai T, Wakimoto N Yin D Gery S, Kawamata N, et al. Histone deacetylase inhibitor, suberoylanilide hydroxamic acid (Vorinostat, SAHA) profoundly inhibits the growth of human pancreatic cancer cells. Int J Cancer. 2007; 121(3):656-665.

[43] Flotho C, Claus R, Batz C, Schneider M, Sandrock I, et al. The DNA methyltransferase inhibitors azacitidine, decitabine and zebularine exert differential effects on cancer gene expression in acute myeloid leukemia cells. Leukemia. 2009; 23(6):1019-1028.

[44] Lemaire M, Momparler LF, Raynal NJ, Bernstein ML, Momparler $\mathrm{RL}$. Inhibition of cytidine deaminase by zebularine enhances the antineoplastic action of 5-aza-2'-deoxycytidine. Cancer Chemother Pharmacol. 2009; 63(3):411-416.

[45] Cheng JC, Weisenberger DJ, Gonzales FA, Liang G, Xu GL, et al. Continuous zebularine treatment effectively sustains demethylation in human bladder cancer cells. Mol Cell Biol. 2004(3): 24:1270-1278.

[46] Momparler RL, Cote S, Mompaler FL, Idaghdour Y. Epigenetic therapy of acute myeloid leukemia using 5-aza-2'-deoxycytidine (decitabine) in combination with inhibitors of histone methylation and deacetylation. Clin Epigenetics. 2014; 6(1):19.
[47] Momparler RL, Idaghdour Y, Marquez VE, Momparler LF. Synergistic antileukemic action of a combination of inhibitors of DNA methylation and histone methylation. Leuk Res. 2012; 36(8):1049-1054.

[48] Sato T, Yang X, Knapper S, White P, Smith BD, et al. FLT3 ligand impedes the efficacy of FLT3 inhibitors in vitro and in vivo. Blood. 2011; 117(12):3286-3293.

[49] Williams CB, Kambhampati S, Fiskus W, Wick J, Dutreix C, et al. Preclinical and phase I results of decitabine in combination with midostaurin (PKC412) for newly diagnosed elderly or relapsed/refractory adult patients with acute myeloid leukemia. Pharmacotherpy. 2013; 33(12):1341-1352.

[50] Li XY, Wu JZ, Cao HX, Ma R, Wu JQ, et al. Blockade of DNA methylation enhances the therapeutic effect of gefitinib in non-small cell lung cancer cells. Oncol Rep. 2013; 29(5):1975-1982.

[51] Lou YF, Zou ZZ, Chen PJ, Huang GB, Li B, et al. Combination of gefitinib and DNA methylation inhibitor decitabine exerts synergistic anticancer activity in colon cancer cells. PLoS One. 2014; 9(5):e97719.

[52] Momparler RL, Dore BT, Momparler LF. Effect of 5-aza-2'-deoxycytidine and retinoic acid on differentiation and c-myc expression in HL-60 myeloid leukemic cells. Cancer Lett. 1990; 54(1-2):21-28.

[53] Garcia-Manero G, Kantariian HM, Sanchez-Gonzalez B, Yang H, Rosner $\mathrm{G}$, et al. Phase 1/2 study of the combination of 5-aza-2'-deoxycytidine with valproic acid in patients with leukemia. Blood. 2006; 108(10): 3271-3279.

[54] Chu BF, Karpenko MJ, Liu Z, Aimiuwu J, Villalona-Calero MA, et al. Phase I study of 5-aza-2'-deoxycytidine in combination with valproic acid in non-small-cell lung cancer. Cancer Chemother Pharmacol. 2013; 71(1):115-121.

[55] Stathis A, Hotte SJ, Chen EX, Hirte HW, Oza AM, et al. Phase I study of decitabine in combination with vorinostat in patients with advanced solid tumors and non-Hodgkin's lymphomas. Clin Cancer Res. 2011; 17(6):1582-1590. (Article)

[56] Shen H, Laird PW. Interplay between the cancer genome and epigenome. Cell. 2013; 153(1):38-55. (Article)

[57] Lemaire M, Chabot GG, Raynal NJ, Momparler LF, Hurtubise A, et al. Importance of dose-schedule of 5-aza-2'-deoxycytidine for epigenetic therapy of cancer. BMC cancer. 2008: 2:8-128.

[58] Karahoca M, Momparler RL. Pharmacokinetic and pharmacodynamic analysis of 5-aza-2'-deoxycytidine (decitabine) in the design of its dose-schedule for cancer therapy. Clin Epigenetics. 2013; 5(1):3.

[59] Le Tourneau C, Lee JJ, Siu LL. Dose escalation methods in phase I cancer clinical trials. J Natl Cancer Inst. 2009; 101(10):708-720.

[60] Cameron EE, Bachman KE, Myöhänen S, Herman JG, Baylin SB. Synergy of demethylation and histone deacetylase inhibition in the re-expression of genes silenced in cancer. Nat Genet. 1999; 21(1):103-107.

[61] Gore SD. Combination therapy with DNA methyltransferase inhibitors in hematologic malignancies. Nat Clin Pract Oncol. 2005: 2(suppl 1):S30-S35.

[62] Oki Y, Aoki E, Issa JP. Decitabine-bedside to bench. Crit Rev Oncol Hematol. 2007; 61(2):140-152.

[63] Cashen AF, Schiller G, O'Donnell MR, Di-Persio JF. Multicenter, phase II study of decitabine for the first-line treatment of older patients with acute myeloid leukemia. J Clin Oncol. 2010; 28(4): 556-561.

[64] Lübbert M, Rüter BH, Claus R, Schmoor C, Schmid M, et al. A multicenter phase II trial of decitabine as first-line treatment for older patients with acute myeloid leukemia judged unfit for induction chemotherapy. Haematologica. 2012; 97(3):393-401.

[65] Klisovic MI, Maghraby EA, Parthun MR, Guimond M, Sklenar AR, et al. Depsipeptide (FR 901228) promotes histone acetylation, gene transcription, apoptosis and its activity is enhanced by DNA methyltransferase inhibitors in AML1/ETO-positive leukemic cells. Leukemia. 2003; 17(2):350-358.

[66] Hurtubise A, Bernstein ML, Momparler RL. Preclinical evaluation of the antineoplastic action of 5-aza-2'-deoxycytidine and different histone deacetylase inhibitors on human Ewing's sarcoma cells. Cancer Cell Int. 2008; 8:10-1186.

[67] Côté S, Momparler RL. Antineoplastic action of all-trans retinoic acid and 5-aza-2'-deoxycytidine on human DLD-1 colon carcinoma cells. Cell Pharmacol. 1995; 2:8.

[68] Li K, Hu C, Mei C, Ren Z, Vera JC, et al. Sequential combination of decitabine and idarubicin synergistically enhances anti-leukemia effect followed by demethylating Wnt pathway inhibitor promoters and downregulating Wnt pathway nuclear target. J Transl Med. 2014; 12:167.

[69] Morita S, Lida S, Kato K, Takagi Y, Uetake $\mathrm{H}$, et al. The synergistic effect of 5-aza-2'-deoxycytidine and 5-fluorouracil on drug-resistant tumors. Oncology. 2005; 71(5-6):437-445.

[70] Blum W, Klisovic RB, Hackanson B, Liu Z, Liu S, et al. Phase I study of decitabine alone or in combination with valproic acid in acute myeloid leukemia. J Clin Oncol. 2007; 25(25):3884-3891.

[71] Kuendgen A, Knipp S, Fox F, Strupp C, Hildebrandt B, et al. Results of a phase 2 study of valproic acid alone or in combination with alltrans retinoic acid in 75 patients with myelodysplastic syndrome and relapsed or refractory acute myeloid leukemia. Ann Hematol. 2005; 84 suppl 1:61-66.

[72] Fang F, Balch C, Schilder J, Breen T, Zhang S, et al. A phase 1 and pharmacodynamic study of decitabine in combination with carboplatin in patients with recurrent, platinum-resistant, epithelial ovarian cancer. Cancer. 2010; 116(17):4043-4053. 